\title{
Phylogenomic Analysis of Campylobacter fetus Reveals a Clonal Structure of Insertion Element ISCfe1 Positive Genomes
}

OPEN ACCESS

Edited by:

Daniel Yero,

Autonomous University of Barcelona

Spain

Reviewed by:

Sabine Kienesberger,

University of Graz, Austria

Min Yue,

Zhejiang University, China

${ }^{*}$ Correspondence:

Mostafa Y. Abdel-Gli

mostafa.abdelgli@@fli.de

Specialty section:

This article was submitted to

Evolutionary and Genomic

Microbiology,

a section of the journa

Frontiers in Microbiology

Received: 20 July 2020 Accepted: 26 October 2020 Published: 12 November 2020

Citation:

Abdel-Glil MY, Hotzel H, Tomaso H and Linde J (2020)

Phylogenomic Analysis of Campylobacter fetus Reveals a Clonal Structure of Insertion Element ISCfe1 Positive Genomes.

Front. Microbiol. 11:585374. doi: 10.3389/fmicb.2020.585374

\author{
Mostafa Y. Abdel-Glil1,2*, Helmut Hotzel ${ }^{1}$, Herbert Tomaso ${ }^{1}$ and Jörg Linde ${ }^{1}$ \\ ${ }^{1}$ Institute of Bacterial Infections and Zoonoses, Friedrich-Loeffler-Institut, Jena, Germany, ${ }^{2}$ Department of Pathology, Faculty \\ of Veterinary Medicine, Zagazig University, Ash Sharqiyah, Egypt
}

Subspecies of the species Campylobacter fetus are associated with specific host niches including mammals and reptiles. Campylobacter fetus subsp. fetus is a zoonotic pathogen infecting humans. Infections can vary from an acute intestinal illness to severe systemic infections, with sheep and cattle as major reservoirs. In contrast, Campylobacter fetus subsp. venerealis causes bovine genital campylobacteriosis, which leads to abortion in cattle and a high economic burden for the farmers. Therefore, highquality molecular subtyping is indispensable for interventional epidemiology. We used whole-genome sequencing (WGS) data of 283 Campylobacter fetus strains from 18 countries and compared several methods for Campylobacter fetus subtyping, including WGS, multilocus sequence typing, PCR assays, and the presence of the insertion element ISCfe1. We identified a highly clonal clade (designated as clade 1) that harbors the insertion sequence ISCfe1. The presence of this insertion sequence is an essential diagnostic tool for the identification of the subspecies Campylobacter fetus subsp. venerealis, serving as a target for several PCR assays. However, we have found a high sequence variability for the ISCfe1 besides the presence of ISCfe1-paralogues in certain other genomes $(n=7)$ which may cause incorrect diagnostic results. Clade 1 seems to be the cattle-specific clade of this species. We propose that only this clade might be designated as Campylobacter fetus subsp. venerealis as it harbors the ISCfe1 marker sequence, which is a major target for molecular methods currently used for Campylobacter fetus subspecies identification. Fostering this proposal, we defined eleven stable nucleotide markers specific for this clade. Additionally, we developed a bioinformatics toolbox for the fast identification of this clade based on WGS data. In conclusion, our results demonstrate that WGS can be used for Campylobacter fetus subtyping overcoming limitations of current PCR and MLST protocols.

Keywords: Campylobacter fetus, ISCfe1, bovine genital campylobacteriosis, WGS, MLST

\section{INTRODUCTION}

The species Campylobacter (C.) fetus comprises three subspecies with distinct clinical significance: C. fetus subsp. fetus $(C f f)$ and $C$. fetus subsp. venerealis $(C f v)$ are both mammal-associated, and C. fetus subsp. testudinum (Cft) occurs in reptiles (Gilbert et al., 2017, 2018). Strains of Cfv are highly niche-specific and restricted to the genital tract of cattle (Silveira et al., 2018). Cfv causes 
bovine genital campylobacteriosis (BGC), a disease associated with abortion and infertility in cattle with significant economic losses (Silveira et al., 2018). In contrast, Cff was found in humans, cattle, sheep, and other animal species. Cff resides as normal flora in the human's intestine (Sprenger et al., 2012; Iraola et al., 2017) but can also cause diarrhea or systemic disease in humans and sporadic abortion in sheep and cattle (Guillermo et al., 2001; Chaillon et al., 2010; Sprenger et al., 2012; Iraola et al., 2017).

Given the distinct host-niche preference and clinical relevance, subspecies differentiation is crucial for disease control and veterinary public health (Schulze et al., 2006). The reptileassociated Cft is divergent from Cfv and Cff (Fitzgerald et al., 2014) with pairwise average nucleotide identity (ANI) of 92\% (Iraola et al., 2017), which is below the commonly accepted ANI for species definition (95\%) (Richter and Rossello-Mora, 2009). Therefore, $C f t$ was proposed as an independent species (Iraola et al., 2017). Cfv and Cff, on the other hand, are very similar at the DNA level (Iraola et al., 2017). These subspecies can be distinguished using biochemical testing based on $1 \%$ glycine tolerance and $\mathrm{H}_{2} \mathrm{~S}$ production in cysteine media (Schulze et al., 2006; Silveira et al., 2018). However, these methods have poor reproducibility and are usually not concordant with molecular methods (Van Der Graaf-Van Bloois et al., 2014). Molecular methods for $C$. fetus subtyping include multilocus sequence typing (MLST) (Van Bergen et al., 2005a), amplified fragment length polymorphisms (AFLP) (Van Bergen et al., 2005b), pulsed-field gel electrophoresis (PFGE) (On and Harrington, 2001), and polymerase chain reaction (PCR) (Wang et al., 2002; Abril et al., 2007; Mcgoldrick et al., 2013; Van Der Graaf-Van Bloois et al., 2013). The latter is valuable for detecting $C f v$ without culturing and includes $C f v$-specific PCRs for detecting ISCfe 1 (Abril et al., 2007; Mcgoldrick et al., 2013; Van Der Graaf-Van Bloois et al., 2013), a parA gene (Hum et al., 1997; Mcmillen et al., 2006) or others targets (Moolhuijzen et al., 2009; Iraola et al., 2012), but also a Cff-specific PCR (Wang et al., 2002).

Germany is currently declared free of bovine genital campylobacteriosis. Nevertheless, each bull used for semen production has to be tested once a year for the presence of C. fetus subsp. venerealis and Tritrichomonas foetus. Only if both tests are negative, the semen may be sold. The present diagnostic methods used for subspecies detection of C. fetus lack reproducibility or are sometimes contradictory. Therefore, this study aimed at using public genomic data of 283 C. fetus strains and in silico comparing molecular methods used to assign the C. fetus subspecies. We further identified new nucleotide markers and developed a bioinformatics tool for C. fetus subtyping using WGS for outbreak investigations.

\section{MATERIALS AND METHODS}

\section{Retrieval and Curation of Public Genomic Data}

We downloaded publicly available genomes of $C$. fetus from NCBI, including assemblies $(n=173)$ and raw reads $(n=327)$ (Supplementary Table S1). In a first step (A), these public data were curated for quality assurance via removing outlier genomes (Figure 1). We excluded genomes that deviated from the average $C$. fetus genome size $(\sim 1.8 \mathrm{Mbp} \pm 25 \%)$, possibly indicating contamination or insufficient DNA sequencing quality. For that, raw reads were assembled using shovill v1.0.4 ${ }^{1}$ (options -minlen 500 -mincov 3). Step B performed species confirmation via measuring pairwise ANI using pyani v0.2.3 (Pritchard et al., 2016) and excluded genomes with $<95 \%$ accordance (Supplementary Table S2). This procedure removed all Cft genomes. Step C removed data redundancies via matching sequences from SRA and assembly databases based on BioSample-ID. In the case that samples were available in both databases, we kept only raw reads. The final data set comprised 283 genomes (Table 1, details Supplementary Table S1). These strains are geographically diverse as they were collected from 18 countries of six continents. Isolates from Europe were the most frequent (41\%) followed by those from North America (32\%) and South America (14\%). The isolates were collected between 1952 and 2018. Many isolates were of bovine origin (37\%) reflecting the preferred ecological niche of this species, followed by human $(26 \%)$ and ovine isolates $(6 \%)$ as well as one isolate from a monkey and two from red-footed tortoises (Chelonoidis carbonarius) (Gilbert et al., 2017, 2018; Iraola et al., 2017).

\section{Whole Genome-Based Bioinformatics Analysis}

For whole-genome comparison, we used snippy $\mathrm{v} 4.6^{2}$ to identify high-quality variants with Illumina reads as input and the genome of strain 82-40 (accession CP000487) as a reference. The default parameters of snippy v4.6 were applied. Assembled genomes were included using the option (-ctgs) in snippy v4.6. For genome clustering, we used hierBAPS (Cheng et al., 2013), which defines clades hierarchically independent from a phylogenetic analysis. As input for hierBAPS, we used recombination filtered and unfiltered core genome alignment. The MATLAB version of hierBAPS was used with two nested clustering levels, and a priori upper bound for clusters number set over the interval between 10 and 100 for 10 independent runs. Gubbins v2.2.1 was used with default settings (Croucher et al., 2015) to remove recombination sites. As input for Gubbins, we constructed a pseudo-whole-genome alignment using snippyclean_full_aln ${ }^{2}$ in which the strains' genome sequences were replaced by the reference genome updated with specific SNPs for each strain (Croucher et al., 2015). The number of SNP variations between genome pairs were calculated using snpdists v0.6.2 $2^{3}$. Consensus SNP sites were extracted using snpsites $\mathrm{v} 2.4 .1^{4}$. To root the phylogenetic tree, we initially included a Cft genome (accession CP027287) and identified two Cff genomes from reptiles that were divergent to the data set. RAxML v8.2.10 (Stamatakis, 2014) was used to construct a maximum likelihood (ML) phylogeny using the GTR $+\Gamma$ nucleotide substitution model, and 100 bootstrap support for bipartitions (options -m ASC_GTRGAMMA -asc-corr = lewis). The ML

\footnotetext{
${ }^{1}$ https://github.com/tseemann/shovill

${ }^{2}$ https://github.com/tseemann/snippy

${ }^{3}$ https://github.com/tseemann/snp-dists

${ }^{4}$ https://github.com/sanger-pathogens/snp-sites
} 


\section{Campylobacter fetus - Taxonomy ID: 196}

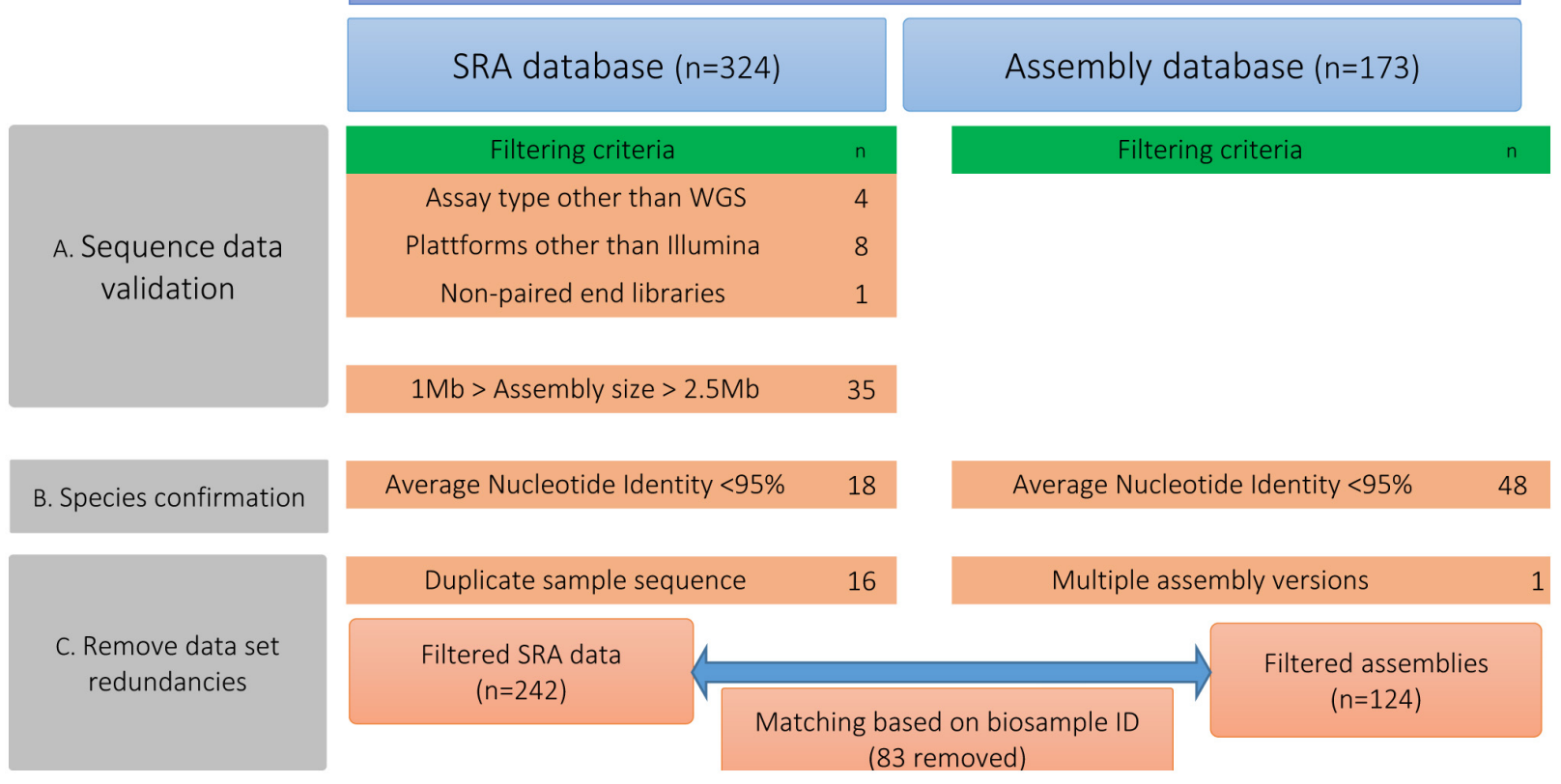

FIGURE 1 | Different steps for filtering the publicly available genomic data of Campylobacter fetus including number of filtered data sets. Step A performed quality assurance via removing genomes that deviated from the average C. fetus genome size ( $1.8 \mathrm{Mbp} \pm 25 \%)$. Step B measured pairwise ANI and excluded genomes with $<95 \%$ accordance. Step C removed data redundancies by matching raw sequence data and genome assemblies based on the BioSample-ID.

tree was visualized using iTOL (Letunic and Bork, 2019) and FigTree v1.4.3 $3^{5}$.

We used whole-genome data to predict MLST types, PCR amplicons, and insertion elements. MLST profiles were determined based on PubMLST database (Jolley and Maiden, 2010) using mlst v2.15.2 ${ }^{6}$ and ARIBA v2.14.4 (Hunt et al., 2017) with default settings. For in silico PCR, we used a PERL script $^{7}$ (accessed 11.2019, default settings) and Geneious prime 2019.2.3 $3^{8}$ with primer sets from previous studies (Hum et al., 1997; Mcmillen et al., 2006; Abril et al., 2007; Mcgoldrick et al., 2013; Van Der Graaf-Van Bloois et al., 2013; Iraola et al., 2016; Supplementary Table S3). Looking for IS elements, we used ISEscan v1.5.4 (Xie and Tang, 2017) to detect IS families. We additionally used the software abricate ${ }^{9}$ to search the genomes through BLAST (options 90\% identity and 30\% overlap) for the presence of IS element "ISCfel" from the IS finder database. The ISCfe1 paralogues were identified based on the detection of multiple BLAST hits with full coverage in the genomes. To show the differences in the location of ISCfe1 paralogues across the chromosomes of the strains, we used progressiveMauve (Darling et al., 2010) for multiple genome alignment with annotated FASTA files as input. This analysis was restricted to the complete

\footnotetext{
${ }^{5}$ https://github.com/rambaut/figtree

${ }^{6}$ https://github.com/tseemann/mlst

${ }^{7}$ https://github.com/egonozer/in_silico_pcr

${ }^{8}$ https://www.geneious.com

${ }^{9}$ https://github.com/tseemann/abricate
}

circularized genomes ( $n=6$ strains) of clade 1 in which multiple copies of ISCfe1 were detected. For sequence analysis of ISCfe1, we firstly parsed the output of abricate ${ }^{9}$ to extract ISCfe1 sequences longer than 1,500 bp. The software MAFFT v7.307 (Katoh and Standley, 2013) aligned the extracted sequences with options (-auto -adjustdirection). We then used MEGA X (Kumar et al., 2018) to report ISCfe1 sequence divergence with all ambiguous positions being removed for each sequence pair (pairwise deletion option). The average nucleotide differences over sequence pairs within and between the ISCfe1 sequence groups were determined using MEGA X (Kumar et al., 2018) with options "compute within/between group mean distance." To identify strict core genes and gene variants in different clades, we used the software Ridom Seqsphere+ v5.1.0 (Junemann et al., 2013) with default settings. The identified genes were tested for the presence of recombination using the Pairwise Homoplasy Index (PHI) statistic (Bruen et al., 2006), as implemented in the PHIPack software (Bruen, 2005).

For the evaluation of the bioinformatics tool "cfvCatch," we assessed its reproducibility using 20 randomly selected samples at different sequencing depths. For that, we used seqtk ${ }^{10}$ to in silico subsample Illumina read sets of the 20 randomly selected C. fetus strains at sequencing depths ranging between $10 \times$ and $100 \times$. The resulting read sets were used as input for the $c f v$ Catch. Statistical values of the assembled genomes were reported using SeqKit (Shen et al., 2016).

\footnotetext{
${ }^{10} \mathrm{https} / /$ github.com/lh3/seqtk
} 
TABLE 1 | Summary of metadata of 283 Campylobacter fetus strains downloaded from public repositories and analyzed in the study.

\begin{tabular}{|c|c|c|c|c|c|c|c|c|c|}
\hline \multicolumn{3}{|c|}{ Isolation geographic source } & \multicolumn{2}{|c|}{ Isolation time range } & \multicolumn{5}{|c|}{ Origin of isolation (No.) } \\
\hline Continent & Country of isolation & n. $(\%)$ & From - To & Missing & Bovine & Human & Ovine & Others & Missing \\
\hline & Argentina & $35(12.4)$ & $1989-2015$ & 4 & 35 & & & & \\
\hline & Uruguay & $5(1.8)$ & $2013-2017$ & & 1 & 3 & 1 & & \\
\hline \multicolumn{2}{|c|}{ North America } & $92(32.5)$ & & & & & & & \\
\hline \multirow[t]{5}{*}{ Europe } & & $117(41.3)$ & & & & & & & \\
\hline & Belgium & $1(0.4)$ & & 1 & 1 & & & & \\
\hline & Czech Republic & $1(0.4)$ & & - & & & & & 1 \\
\hline & France & $39(14.8)$ & $2004-2014$ & 1 & 1 & 38 & & & \\
\hline & Germany & $19(6.7)$ & $1999-2014$ & - & 16 & & 2 & Monkey (1) & \\
\hline \multirow[t]{4}{*}{ Asia } & & $19(6.7)$ & & & & & & & \\
\hline & India & $1(0.4)$ & 2013 & & & 1 & & & \\
\hline & Taiwan & $17(6)$ & $2002-2014$ & & & 17 & & & \\
\hline & Turkey & $1(0.4)$ & 2013 & & & 1 & & & \\
\hline \multirow[t]{3}{*}{ Oceania } & & $4(1.4)$ & & & & & & & \\
\hline & New Zealand & $1(0.4)$ & 2014 & & & & 1 & & \\
\hline & Australia & $3(1.1)$ & $1964-1967$ & 1 & 2 & & & & 1 \\
\hline \multirow[t]{2}{*}{ Africa } & & $2(0.7)$ & & & & & & & \\
\hline & South Africa & $2(0.7)$ & $2006-2007$ & & 2 & & & & \\
\hline Missing & & $9(3.2)$ & & & & 1 & 2 & & 6 \\
\hline
\end{tabular}

\section{RESULTS AND DISCUSSION}

A recent study by Iraola et al. (2017) classified a global collection of 182 C. fetus strains into eight phylogenetic lineages. The data of Iraola et al., ( $n=169$ out of 182 genomes) were combined with 114 additional genomes from independent studies as well as sequence data from the NCBI (Table 1, details Supplementary Table S1). Based on this extended data set $(n=283$ strains), we identified 18,793 high-quality SNPs that were present in a clonal frame (non-recombinant SNPs) in 281 genomes, while two genomes (GCF_000174675 [Azul94] and SRR6377517 [PNUSAC001504]) were unsuitable for recombination and phylogenetic analysis because of the high percentage of missing/ambiguous nucleotides ( $>25 \%$ ). Two reptilian C. fetus were very distant to other C. fetus from mammals with average ANI $\sim 97.8 \%$. In contrast, mammalian C. fetus harbored highly homogenous genomes with $>99.2 \%$ nucleotide similarity (Supplementary Table S2 and Figure 2). Based on SNP architecture in the core genome of the strains, we concluded the clade structure in a phylogeny-dependent (using a maximum likelihood tree) and independent manner (with hierBAPS). The 281 genomes were classified into eight clades including 279 genomes. In addition, two genomes were assigned as singletons (ERR976359 [2006 $479 \mathrm{~h}$ ] and GCF_007723545 [17144]) (Figure 2 and Supplementary Table S3). The size of the eight clades varied between two and 100 genomes and the number of SNP sites detected for the clades were as follow: 687 SNP sites in clade 1 [ $n=100$ genomes], 865 SNP sites in clade 2 [ $n=76$ genomes], 226 SNP sites in clade 3 [ $n=10$ genomes], 352 SNP sites in clade 4 [ $n=22$ genomes], 112 SNP sites in clade 5 [ $n=12$ genomes], 444 SNP sites in clade 6 [ $n=16$ genomes], 548 SNP sites in clade 7 [ $n=42$ genomes] and 3 SNP sites in clade 8 [ $n=2$ genomes]. These results were in good congruence with previous studies. However, we used a larger data set and a different methodology to report core genome SNPs, confirming the high clonality of $C$. fetus strains. In addition, clade 1 strains (as found in this and previous studies) were restricted to cattle except one strain from a sheep and ten strains of unknown origin (Figure 2). The other clades (2-8) comprised strains isolated from different hosts, including humans, sheep, cattle, and others (Figure 2). Using this global reference phylogeny, we in silico compared molecular methods used for the subtyping of C. fetus.

\section{The Presence of the ISCfe1 Insertion Sequence and Its Sequence Variability}

ISCfe1 has been reported as a specific marker for Cfv (Abril et al., 2007; Mcgoldrick et al., 2013). We searched the genomes through BLAST for the ISCfe 1 with thresholds: $90 \%$ identity and 


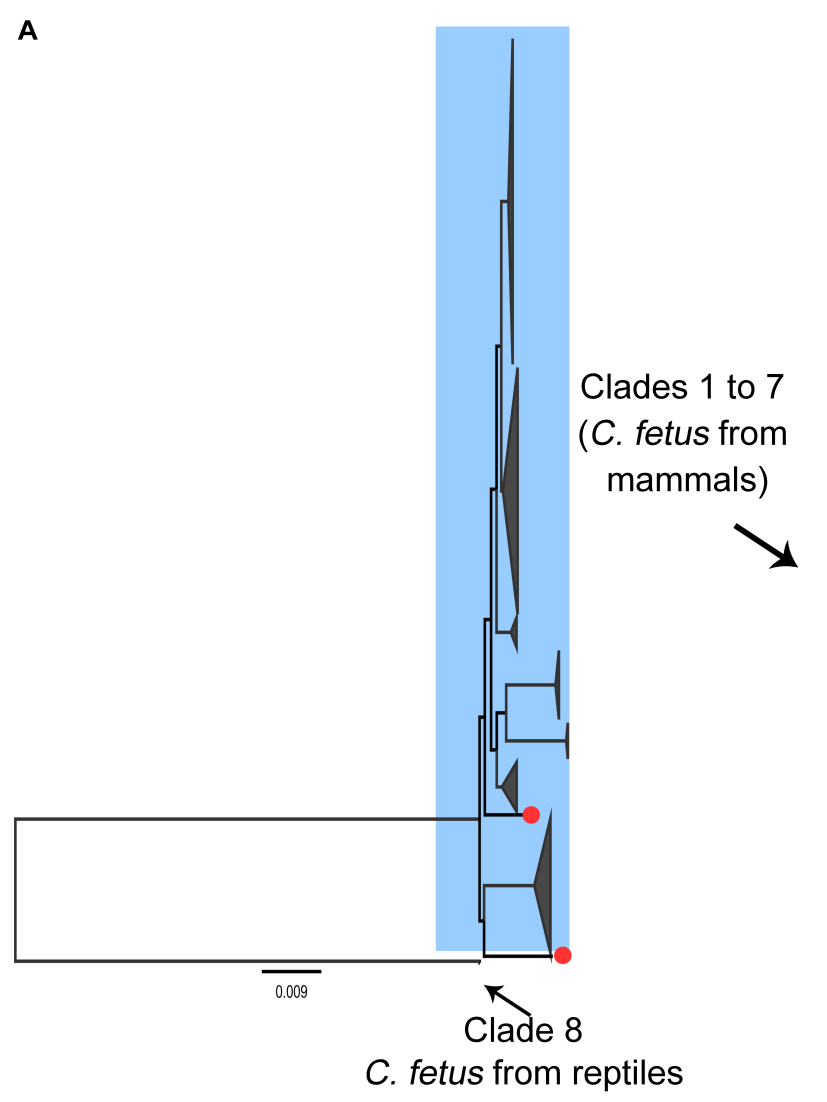

B

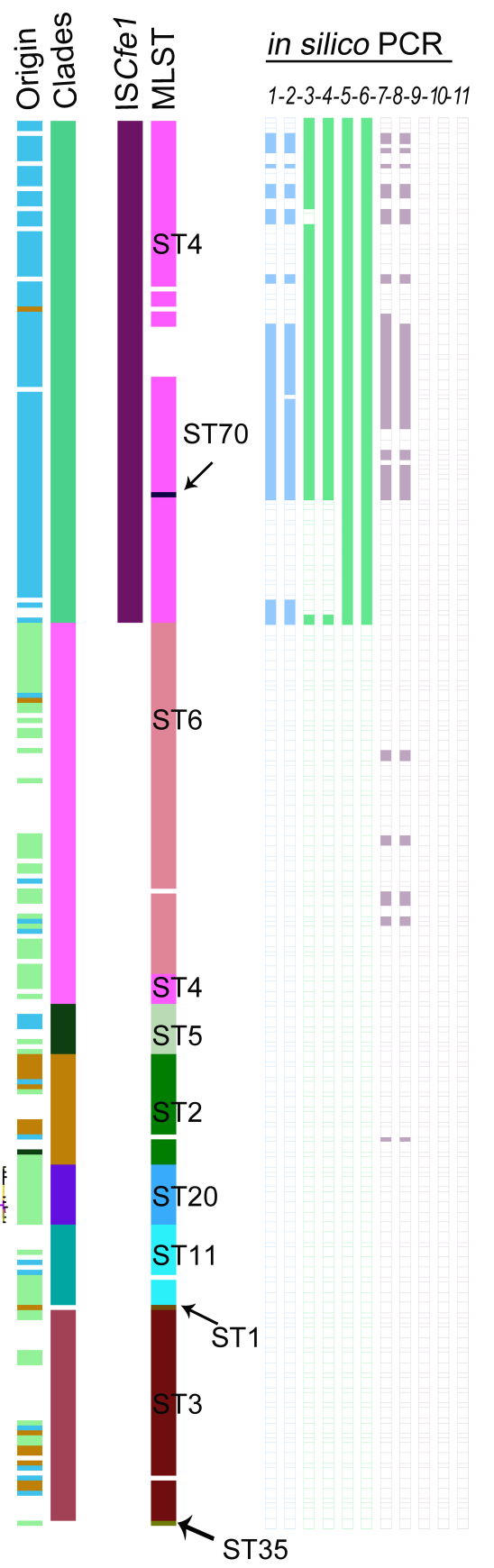

\begin{tabular}{|c|c|c|}
\hline Phylogenetic clades & Origin of isolates & ISCfe1 \\
\hline Clade 1 & Cattle & Present \\
\hline Clade 2 & Human & Absent \\
\hline Clade 3 & Sheep & \\
\hline Clade 4 & Monkey & \\
\hline Clade 5 & Unknown & \\
\hline Clade 6 & & \\
\hline Clade 7 & & \\
\hline
\end{tabular}

\title{
in silico PCR (Cfv-specific)
}

1. parA-Hum et al., 1997

2. parA-McMillen et. al., 2006

3. ISCfe1-Abril et al., 2007

4. ISCfe1 (ISC1)-van der Graaf et al., 2013

\author{
5. ISCfe1 (ISC2)-van der Graaf et al., 2013 \\ 6. ISCfe1-McGoldrick et al., 2013 \\ 7. virB11-Iraola et al., 2012 \\ 8. virB6-Moolhuijzen et al., 2009
}

9. virD4-Moolhuijzen et al., 2009 10 virB4-Moolhuijzen et al., 2009 11. van Bergen et al., 2005

FIGURE 2 | Phylogenetic structure of Campylobacter fetus genomes based on 18,793 high-quality SNPs that were present in a clonal frame in the 281 C. fetus genomes. (A) A maximum likelihood phylogenetic tree with the eight major clades of the species being collapsed. Red colored tips indicate two strains regarded as singletons. Note that clade 8 includes two Campylobacter fetus genomes from reptiles which were divergent to mammalian C. fetus genomes (ANI 97.8\%). (B) A maximum likelihood phylogenetic tree showing clades 1 to 7 . The bootstrap values are indicated with branch coloring according to the legend provided in the figure, 0 to $100 \%$ bootstrap support corresponds to the branch colors from black to violet, using yellow as the midpoint. Columns beside the phylogenetic tree correspond, respectively, to host of isolation, phylogenetic clades, the presence of ISCfe1 element, the MLST types, and the results of in silico PCR (numbered one to eleven according to the primer used as in legends). White spaces in the PCR indicate negative results while in MLST, they indicate no assignment to known MLST type. 

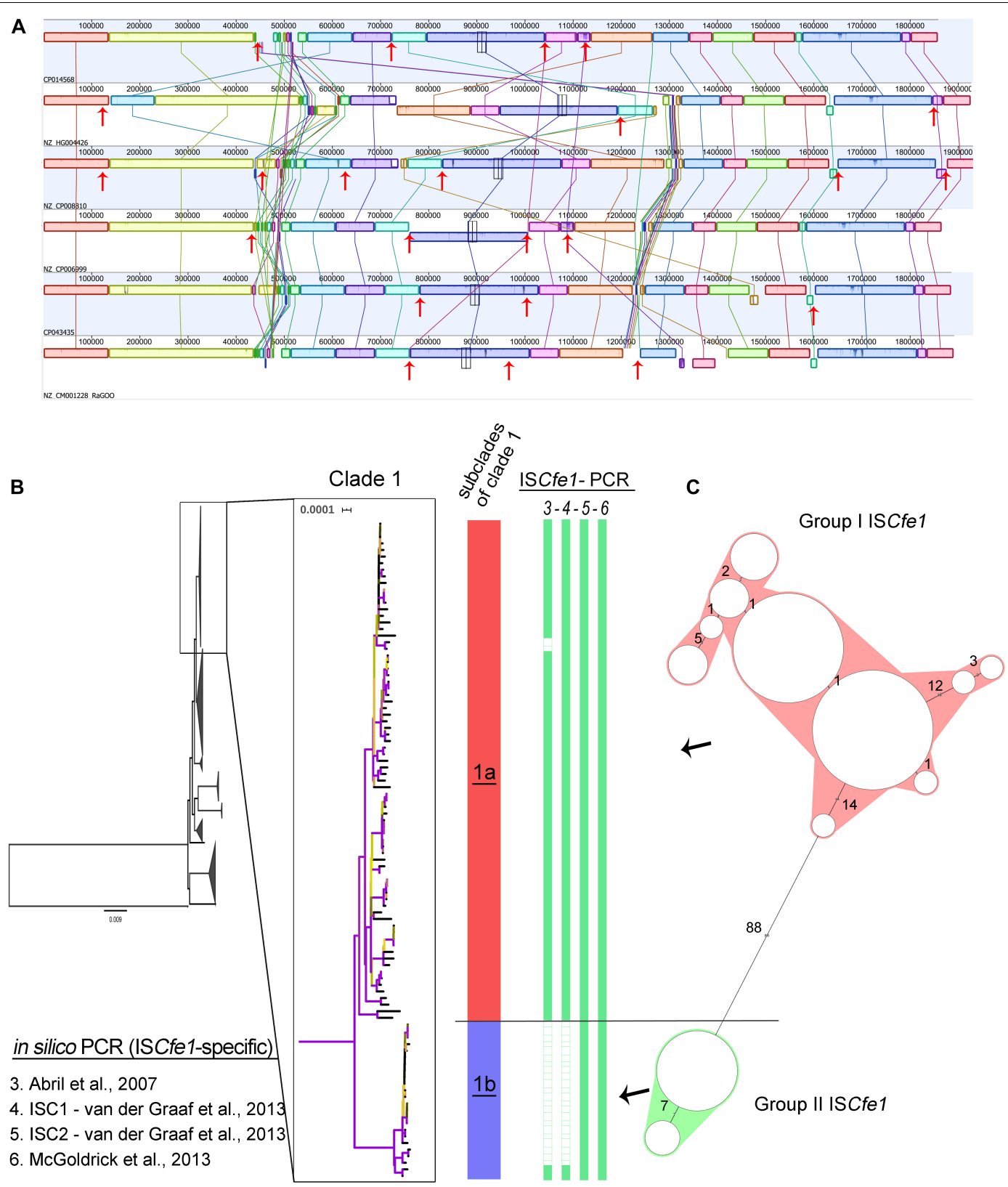

FIGURE 3 | The presence of the ISCfe1 insertion sequence and its sequence variability. (A) Mauve alignment of the chromosomes from the six complete Campylobacter fetus genomes in clade 1 showing the location of the ISCfe 1 paralogues across the chromosomes (red arrows) and their frequent association with inversions and shifts of the chromosomal locally collinear blocks. (B) A close-up view of the phylogenetic clade 1 shows two distinct subclades (1a and $1 \mathrm{~b}$ ). Two ISCfe 1 groups based on the cluster analysis of the ISCfe1 sequences (C) were reflected by the phylogenetic structure of clade 1 . Group I ISCfe1 is present in the subclade "1a" while group II of ISCfe1 is present in subclade "1b".

$30 \%$ overlap. The results showed that this marker element exists exclusively in all clade 1 genomes (Figures 2, 3).

Further analysis of the ISCfe1 identified the presence of multiple copies (ISCfe1 paralogues) in certain genomes comprising the six complete genomes in clade 1 (Figure 3A) as well as one draft genome. Three to eight copies per genome were present (Supplementary Tables S3, S4). In the complete genomes, the ISCfe1 paralogues were distributed on the strains' chromosome (3 - 6 copies per chromosome) and plasmids (1 - 3 copies per plasmid; Supplementary Table S4). The average identity of the ISCfe1 paralogues within the same genome varied between 98 and 100\% (Supplementary Table S4). The positions of the ISCfe1 across the chromosomes are shown in Figure 3A. Interestingly, ISCfe1 was located at the boundaries of several locally collinear blocks in the chromosome. Some inversions 
of the chromosomal blocks were also bordered by this insertion sequence.

It is noteworthy to mention that the failed detection of the ISCfe 1 paralogues in most of the investigated genomes might be due to their fragmented status as the majority of the data were sequenced using short-read sequencing methods. These technologies may not be adequate tools for the analysis of repeats. These facts hinder the full assembly of ISCfe1 and explain our relaxed BLAST coverage cut-off (>30\%).

In order to investigate the overall sequence variability of ISCfe1, we compared sequences from 123 ISCfe1 in which $>70 \%(1,500 \mathrm{bp})$ of the sequence was obtained (Figure 3C and Supplementary Figure S1). 87 sequences were from strains with single copies and 35 sequences from seven strains with up to eight copies. Based on the sequence divergence of ISCfe1, two stable and divergent groups exist (Figure 3C and Supplementary Figure S1). The mean SNP differences of 101 ISCfel sequences within group I was $1.73 \mathrm{SNPs}(P$ distance $=0.003)$. The mean SNP differences of 22 ISCfe1 sequences within group II was 6.39 SNPs $(P$ distance $=0.001)$. The mean SNP differences between both groups was $124.43 \mathrm{SNPs}(P$ distance $=0.06)$. Interestingly, the two ISCfe1 groups were reflected by the phylogenetic structure of clade 1 (Figure 3B), as group I ISCfe 1 clustered together in a subclade of clade 1 , which is distant by 123 different genes to subclade 2. Subclade 2 harbors the group II of ISCfe1, exclusively.

\section{MLST for Campylobacter fetus Subtyping}

Multilocus sequence typing grouped the 283 strains into eleven sequence types (STs; Figure 2 and Supplementary Table S3). The most frequent MLST types in this study were ST4 $(n=93)$ and ST6 $(n=70)$. Seventeen strains were not assigned to known STs (Supplementary Table S3).

In clade 1, 88 out of 100 genomes were assigned to known STs, including 87 genomes of ST4 and one genome of ST70 (ERR1046003 [INTA19]). Twelve genomes were not reported to known STs. This includes one genome (ERR1203904 [UK13]) with a new variant (G259A) for the gltA gene as well as 11 genomes ( 10 of them form a cluster) that had $85-88 \%$ coverage of the aspA gene. In an attempt to improve the coverage for this aspA gene, we used FASTQ reads and directly mapped them against the aspA gene (Hunt et al., 2017). However, this has not improved the coverage pattern across the gene.

The genomes of clade 2 were assigned to ST6 except seven genomes that comprise one genome (ERR976358 [2006-367 h]) with a new variant (G222A) for the uncA gene as well as six genomes of ST4 (Figure 2 and Supplementary Table S3). The ST4 strains in clade 2 were previously reported in humans (Iraola et al., 2015), while two were of unknown origin. This is in contrast to ST4 in clade 1, which was reported in cattle. Interestingly, ST6 was just one allele distant to ST4. Similarly, clade 2 was very closely related to clade 1 .

The phylogenetic clades 3 to 8 showed congruent results with MLST, in which clades 3, 5, and 8 corresponded to ST5, ST20, and ST69, respectively (Figure 2 and Supplementary Table S3). One strain in each of clades 4, 6 and 7 was not reported to known MLST type due to insufficient coverage to the MLST genes or due to the presence of gaps. The remaining strains of clade 4,6 , and 7 comprised ST2 $(n=21)$, ST11 $(n=15)$ and ST3 $(n=41)$, respectively.

In summary, MLST showed good agreement with the WGSbased phylogenetic clades, but WGS could resolve inconsistencies associated with bovine and human ST4 isolates. The ST4 was previously reported as $C f v$-associated ST (Van Bergen et al., 2005a; Silveira et al., 2018) along with ST7 and ST12 (Van Bergen et al., 2005a). The latter were not detected in this study.

\section{In silico PCR Assays for Campylobacter fetus Subtyping}

We tested 17 primer pairs for C. fetus including primer pairs from five PCR assays to detect C. fetus, one PCR to detect Cff (Wang et al., 2002), and 11 PCR assays to detect Cfv. Many PCR assays for the identification of $C f v$ target the ISCfe1 (Abril et al., 2007; Mcgoldrick et al., 2013; Van Der Graaf-Van Bloois et al., 2013), a parA gene (Hum et al., 1997; Mcmillen et al., 2006), but some assays have other targets (Van Bergen et al., 2005b; Moolhuijzen et al., 2009; Iraola et al., 2012; Figure 2 and Supplementary Table S3).

Results showed a high sensitivity of primer pairs that detect C. fetus at the species level, with primers developed by Mcgoldrick et al. (2013) being 100\% sensitive. The primers reported by Abril et al. (2007) did not detect genomes that belong to clade 4 and clade 8 , as well as some clade 1 genomes.

At the subspecies level, the Cff-specific PCR assay reported by Wang et al. (2002) was $32 \%$ sensitive to clade 2, 6, and 7, while genomes of the clades $1,3,4,5$, and 8 were all negative. On the other hand, the $C f v$-specific PCR assays showed positive results only for clade 1 genomes, except one PCR primer-pair previously described by Moolhuijzen et al. (2009) and Iraola et al. (2012) that was also positive for genomes of clade 2 ( $n=8$ genomes) and clade 4 ( $n=1$ genome) (Figure 2 and Supplementary Table S3).

The ISCfe1 specific primer described by Mcgoldrick et al. (2013) was 100\% sensitive to all genomes of clade 1 (Figures 2, 3 and Supplementary Table S3). One primer set (ISC2) described by Van Der Graaf-Van Bloois et al. (2013) was also 100\% sensitive to clade 1 genomes (Figures 2, 3 and Supplementary Table S3). Nevertheless, ISCfe1 primers from Abril et al. (2007) as well as one primer set (ISC1) from Van Der Graaf-Van Bloois et al. (2013) could not detect most of group II ISCfe1-carrying strains $(n=22$ strains, Figures 2, 3 and Supplementary Table S3). The binding site of latter primer pairs located within a variable sequence region between the ISCfe1 group I and group II (Supplementary Figure S2). In contrast, PCR primers for the parA gene described by Hum et al. (1997) and Mcmillen et al. (2006) detected only 55 and $52 \%$ of clade 1 genomes, respectively. These results were confirmed using BLAST search (90\% identity) for the parA gene (CP006999.2:1246091-1246231) in which we found the gene only in $55 \%$ of clade 1 genomes. We, therefore, concluded that ISCfel-positive genomes do not necessarily carry the parA gene explaining the lack of congruence for different PCRs to detect $C f v$ (Figure 2 and Supplementary Table S3). Furthermore, the in silico evaluation of C. fetus-specific PCRs 
TABLE 2 | Eleven characteristic nucleotide markers for clade I in comparison to other clades.

\begin{tabular}{|c|c|c|c|c|c|c|c|c|c|c|}
\hline $\begin{array}{l}\text { Nucleotide Position } \\
\text { in reference genome }\end{array}$ & Locus tag & Protein ID & $\begin{array}{l}\text { Gene } \\
\text { Length }\end{array}$ & Gene & Product & $\begin{array}{c}\text { Cfv } \\
(n=100)\end{array}$ & $\begin{array}{c}\text { Cff } \\
(n=182)\end{array}$ & $\begin{array}{l}\text { Effect to } \\
\text { reference } \\
\text { sequence }\end{array}$ & Cfv AA & Cff AA \\
\hline 39784 & CFF8240_0048 & ABK83415.1 & 396 & rpsH & ribosomal protein S8 & T & $\mathrm{C}$ & synonymous & L & L \\
\hline 343646 & CFF8240_0373 & ABK82476.1 & 552 & & conserved hypothetical protein & T & C & synonymous & L & L \\
\hline 399029 & CFF8240_0424 & ABK81969.1 & 708 & & conserved hypothetical protein & C & T & synonymous & G & $G$ \\
\hline 535915 & CFF8240_0528 & ABK83346.1 & 1131 & & $\begin{array}{l}\text { integral membrane } \\
\text { protein-permease component, } \\
\text { involved in lipoprotein release }\end{array}$ & T & C & synonymous & S & $S$ \\
\hline 654050 & CFF8240_0641 & ABK82108.1 & 507 & & Hit family protein & A & G & non-synonymous & I & V \\
\hline 979346 & CFF8240_0977 & ABK82797.1 & 747 & & $\begin{array}{l}\text { NADP-dependent } \\
\text { I-serine/l-allo-threonine } \\
\text { dehydrogenase ydfg }\end{array}$ & C & $\mathrm{T}$ & non-synonymous & C & Y \\
\hline 1020607 & CFF8240_1016 & ABK82995.1 & 879 & & phosphatase, Ppx/GppA family & A & C & non-synonymous & D & E \\
\hline 1025195 & CFF8240_1023 & ABK83085.1 & 615 & & translocator protein, LysE family & C & T & non-synonymous & S & $\mathrm{N}$ \\
\hline 1359201 & CFF8240_1380 & ABK83386.1 & 579 & & $\begin{array}{l}\text { general glycosylation pathway } \\
\text { protein }\end{array}$ & C & $\mathrm{T}$ & non-synonymous & M & । \\
\hline 1438083 & CFF8240_1456 & ABK82820.1 & 1032 & hemE & $\begin{array}{l}\text { uroporphyrinogen } \\
\text { decarboxylase }\end{array}$ & A & $\mathrm{G}$ & synonymous & D & $\mathrm{D}$ \\
\hline 1452736 & CFF8240_1473 & ABK83285.1 & 1791 & lepA & GTP-binding protein LepA & G & A & synonymous & T & $\mathrm{T}$ \\
\hline
\end{tabular}


indicates that the primer pairs described by Mcgoldrick et al. (2013) performed best at the species and subspecies levels. This is in contrast to the updated international standards for the BGC at the OIE Terrestrial Manual (2018) (OIE) in which Abril et al. (2007) showed the highest sensitivity (97\%) to detect Cfv. Yet, based on our analysis, this PCR assay was only $78 \%$ sensitive and could not detect 22 genomes with ISCfe1 group II of the investigated dataset.

Moreover, the sequence divergence (two groups) and repetition (multiple copies within the genomes) of ISCfe1 may hinder its correct identification with PCR assays as described (Van Der Graaf-Van Bloois et al., 2013). The results of in silico PCR may substantially differ from in vitro PCRs due to differences in primer binding. In addition, the fragmentation of most of the investigated genomes may negatively influence the output of in silico PCR. This can also be considered as a limitation of this study.

\section{New Markers and a Bioinformatics Tool for Campylobacter fetus Subtyping}

We determined group-specific nucleotide markers for the eight defined clades, which could be investigated in the new strains for fast clade delineation without the need to reconstruct the whole phylogeny. These nucleotide markers were defined as specific nucleotide variants, which at a specific position, are identical in all genomes of one specific clade and different from all genomes of all other clades, ignoring indels and variants located in the intergenic regions or within repeated sequences (Supplementary Table S5).

Given the clinical importance of clade 1 being the cattle specific clade of this species, we identified eleven stable nucleotide markers specific for this clade, consequently for the subspecies Cfv (Table 2). The eleven SNPs comprised six synonymous and five non-synonymous variants and were identified within the most stable and strict core genes in all strains. The genes exhibited very limited nucleotide diversity with 4 to 13 variants observed for the eleven genes. These genes apparently have not recombined (non-significant PHI test) or had detrimental mutations that might have resulted in internal stop codons.

We then developed " $c f v C a t c h " 11$, a bioinformatics toolbox that fetches genomic data of $C$. fetus (Figure 4) and reports nucleotide markers specific for clade $1(C f v)$ and markers specific for clades 2-8 (Cff). In addition, $c f v C a t c h$ reports MLST types

${ }^{11}$ https://gitlab.com/FLI_Bioinfo/cfvcatch

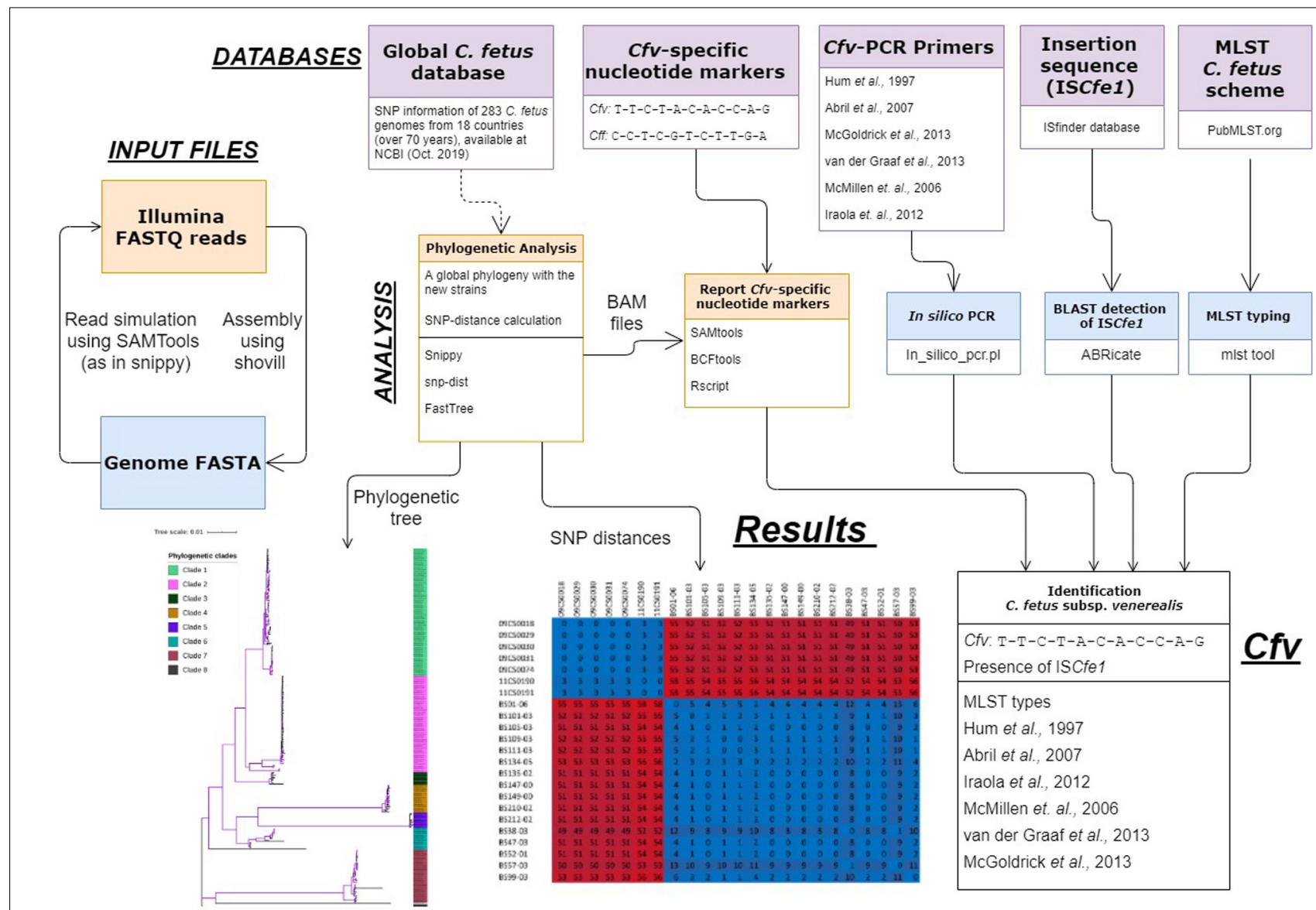

FIGURE 4 | An overview of the cfvCatch workflow to identify the Campylobacter fetus subsp. venerealis (Cfv) strains. 
and the presence of ISCfe1 as well as results of in silico PCR assays. Nevertheless, to report subspecies, $c f v$ Catch takes only into account the phylogenetic position of $C$. fetus strains and the presence of the ISCfel. Strains are characterized as $C f v$ if they (1) harbor the insertion element ISCfe1 and (2) have the nucleotide variants specific for clade 1 (T-TC-T-A-C-A-C-C-A-G), while strains that (1) do not harbor the insertion element ISCfe1 and (2) have the nucleotide variants specific for clades 2-8 (C-C-T-C-G-T-C-T-T-G-A), are reported as $C f f$. This assumption has been applied to 283 C. fetus genomes that represent diverse strains of global distribution (Supplementary Table S1) and sequenced at various read lengths using Illumina platforms. We further integrated the typing results of this global data set in $c f v$ Catch for future phylogenetic comparison of new strains. The current implementation of the tool makes use of sequencing read mapping to a reference genome, therefore FASTQ reads are required as input. This is the only mandatory parameter for this software.

We assessed the performance of cfvCatch at different sequencing depths $(10 \times$ to $200 \times)$ using an exemplary data set of $20 C f v$ strains. The results showed that at a coverage depth of more than $30 \times$, the subspecies detection was highly reproducible. However, the coverage depth of $10-20 \times$ resulted in failure in subspecies assignment for two (out of 20) genomes (Supplementary Table S6).

\section{CONCLUSION}

In this study, we in silico compared molecular methods for C. fetus subspecies identification to the global reference phylogeny. As shown here and in previous studies (in vitro) (OIE; Van Der Graaf-Van Bloois et al., 2013, 2014), results from current PCR protocols were not completely congruent and some of the ISCfel-specific PCRs did not detect most of the group II ISCfe1 (Abril et al., 2007; Van Der GraafVan Bloois et al., 2013). MLST provided good discrimination as ST4 correlated with Cfv strains. However, Cff diagnosed as the causative pathogen for bacteraemia in some human cases (Iraola et al., 2015) also belonged to ST4. Additionally, we found different STs for strains that carry ISCfe1 and were positive in $C f v$-PCR assays. These findings underline the lack of congruence for PCRs and MLST for C. fetus subtyping. It is noteworthy that the most common PCR system for Cff definition relies on the negative output of a $C f v$-selective PCR assay. Negative samples for this PCR are regarded as Cff. This system of differentiation can lead to falsepositive results.

Whole-genome analysis provides a very high resolution and can be reliably used to identify closely related strains for outbreaks and surveillances (Ardelean et al., 2019; Hsu et al., 2020). Whole-genome comparison defines a clonal structure (clade 1) for the ISCfe1-carrying genomes. This clade was previously described by Iraola and colleagues and was identified as the cattle-specific clade of C. fetus in concordance with the epidemiology of $C f v$. In addition, ISCfe 1, which is considered to be the Cfv marker sequence, was exclusively present in this clade. Furthermore, the molecular methods for subtyping the C. fetus, including MLST and PCRs, are not congruent, but showed positive results for clade 1 , only.

In conclusion, we reinforce the importance of the clade structure initially described by Iraola and colleagues in 2017 for molecular subtyping of the C. fetus by adding 114 additional genomes. The strains of clade 1 harbor ISCfe 1 and correspond to the subspecies $C f v$, while strains of clades 2- 8 correspond to Cff, which do not harbor the ISCfe1 element. A PCR targeting ISCfe1 is recommended for subtyping C. fetus. In addition, we defined eleven nucleotide variants as specific markers for clade 1 and developed a bioinformatics toolbox available at https:/gitlab.com/FLI_ Bioinfo/cfvcatch for the fast identification and subtyping of C. fetus using whole genome sequence data based on the recently described clade designation.

\section{DATA AVAILABILITY STATEMENT}

The original contributions presented in the study are included in the article/Supplementary Material, further inquiries can be directed to the corresponding author.

\section{AUTHOR CONTRIBUTIONS}

MA-G designed the study, performed analysis and wrote the manuscript. HH, HT, and JL supervised the work and contributed to the interpretation of the data. All authors contributed to the drafting of the manuscript.

\section{ACKNOWLEDGMENTS}

We thank Kerstin Cerncic, Anja Hackbart, and Byrgit Hofmann at the Institute of Bacterial Infections and Zoonoses, FriedrichLoeffler-Institut, for their excellent assistance.

\section{SUPPLEMENTARY MATERIAL}

The Supplementary Material for this article can be found online at: https://www.frontiersin.org/articles/10.3389/fmicb. 2020.585374/full\#supplementary-material

Supplementary Figure 1 | Cluster analysis of the ISCfe1 sequences showing the ISCfe1 sequence divergence as represented by a minimum spanning tree. Figure was created using BioNumerics version 6.6, in which the ISCfe1 sequences were treated as categorical character data, with a priority rule type set to "number of n-locus variants."

Supplementary Figure 2 | Alignment of the two sequence groups of ISCfe1 with highlighted primer positions. 


\section{REFERENCES}

Abril, C., Vilei, E., Brodard, I., Burnens, A., Frey, J., and Miserez, R. (2007). Discovery of insertion element ISCfe1: a new tool for Campylobacter fetus subspecies differentiation. Clin. Microbiol. Infect. 13, 993-1000. doi: 10.1111/ j.1469-0691.2007.01787.x

Ardelean, A., Calistri, P., Giovannini, A., Garofolo, G., Di Pasquale, A., Conte, A., et al. (2019). Development of food safety risk assessment tools based on molecular typing and WGS of Campylobacter jejuni genome. EFSA J. 17:e170903.

Bruen, T. (2005). PhiPack: PHI Test and other Tests of Recombination. Montreal: McGill University.

Bruen, T. C., Philippe, H., and Bryant, D. (2006). A simple and robust statistical test for detecting the presence of recombination. Genetics 172, 2665-2681. doi: 10.1534/genetics.105.048975

Chaillon, A., Baty, G., Lauvin, M. A., Besnier, J. M., Goudeau, A., and Lanotte, P. (2010). Campylobacter fetus subspecies fetus spondylodiscitis. J. Med. Microbiol. 59, 1505-1508. doi: 10.1099/jmm.0.023382-0

Cheng, L., Connor, T. R., Siren, J., Aanensen, D. M., and Corander, J. (2013). Hierarchical and spatially explicit clustering of DNA sequences with BAPS software. Mol. Biol. Evol. 30, 1224-1228. doi: 10.1093/molbev/mst028

Croucher, N. J., Page, A. J., Connor, T. R., Delaney, A. J., Keane, J. A., Bentley, S. D., et al. (2015). Rapid phylogenetic analysis of large samples of recombinant bacterial whole genome sequences using Gubbins. Nucleic Acids Res. 43:e15. doi: 10.1093/nar/gku1196

Darling, A. E., Mau, B., and Perna, N. T. (2010). progressiveMauve: multiple genome alignment with gene gain, loss and rearrangement. PLoS One 5:e11147. doi: 10.1371/journal.pone.0011147

Fitzgerald, C., Tu, Z. C., Patrick, M., Stiles, T., Lawson, A. J., Santovenia, M., et al. (2014). Campylobacter fetus subsp. testudinum subsp. nov., isolated from humans and reptiles. Int. J. Syst. Evol. Microbiol. 64, 2944-2948. doi: 10.1099/ ijs.0.057778-0

Gilbert, M. J., Duim, B., Timmerman, A. J., Zomer, A. L., and Wagenaar, J. A. (2017). Whole genome-based phylogeny of reptile-associated Helicobacter indicates independent niche adaptation followed by diversification in a poikilothermic host. Sci. Rep. 7:8387.

Gilbert, M. J., Duim, B., Van Der Graaf-Van Bloois, L., Wagenaar, J. A., and Zomer, A. L. (2018). Homologous recombination between genetically divergent Campylobacter fetus lineages supports host-associated speciation. Genome Biol. Evol. 10, 716-722. doi: 10.1093/gbe/evy048

Guillermo, V., Beatriz, G., Dolores, D. M., Asuncion, D. V., Luis, O., and Pedro, D. L. I. (2001). Campylobacter fetus subspecies fetus bacteremia associated with chorioamnionitis and intact fetal membranes. Scand. J. Infect. Dis. 33, 126-127. doi: 10.1080/003655401750065517

Hsu, C.-H., Harrison, L., Mukherjee, S., Strain, E., Mcdermott, P., Zhang, Q., et al. (2020). Core genome multilocus sequence typing for food animal source attribution of human Campylobacter jejuni infections. Pathogens 9:532. doi: 10.3390/pathogens 9070532

Hum, S., Quinn, K., Brunner, J., and On, S. L. (1997). Evaluation of a PCR assay for identification and differentiation of Campylobacter fetus subspecies. Aust. Vet. J. 75, 827-831. doi: 10.1111/j.1751-0813.1997.tb15665.x

Hunt, M., Mather, A. E., Sánchez-Busó, L., Page, A. J., Parkhill, J., Keane, J. A., et al. (2017). ARIBA: rapid antimicrobial resistance genotyping directly from sequencing reads. Microb. Genom. 3:e000131.

Iraola, G., Betancor, L., Calleros, L., Gadea, P., Algorta, G., Galeano, S., et al. (2015). A rural worker infected with a bovine-prevalent genotype of Campylobacter fetus subspecies fetus supports zoonotic transmission and inconsistency of MLST and whole-genome typing. Eur. J. Clin. Microbiol. Infect. Dis. 34, 15931596. doi: 10.1007/s10096-015-2393-y

Iraola, G., Forster, S. C., Kumar, N., Lehours, P., Bekal, S., García-Peña, F. J., et al. (2017). Distinct Campylobacter fetus lineages adapted as livestock pathogens and human pathobionts in the intestinal microbiota. Nat. Commun. 8:1367.

Iraola, G., Hernandez, M., Calleros, L., Paolicchi, F., Silveyra, S., Velilla, A., et al. (2012). Application of a multiplex PCR assay for Campylobacter fetus detection and subspecies differentiation in uncultured samples of aborted bovine fetuses. J. Vet. Sci. 13, 371-376. doi: 10.4142/jvs.2012.13.4.371

Iraola, G., Pérez, R., Betancor, L., Marandino, A., Morsella, C., Méndez, A., et al. (2016). A novel real-time PCR assay for quantitative detection of Campylobacter fetus based on ribosomal sequences. BMC Vet. Res. 12:286. doi: 10.1186/s12917016-0913-3

Jolley, K. A., and Maiden, M. C. J. (2010). BIGSdb: scalable analysis of bacterial genome variation at the population level. BMC Bioinformatics 11:595. doi: 10 . 1186/1471-2105-11-595

Junemann, S., Sedlazeck, F. J., Prior, K., Albersmeier, A., John, U., Kalinowski, J., et al. (2013). Updating benchtop sequencing performance comparison. Nat. Biotechnol. 31, 294-296. doi: 10.1038/nbt.2522

Katoh, K., and Standley, D. M. (2013). MAFFT multiple sequence alignment software version 7: improvements in performance and usability. Mol. Biol. Evol. 30, 772-780. doi: 10.1093/molbev/mst010

Kumar, S., Stecher, G., Li, M., Knyaz, C., and Tamura, K. (2018). MEGA X: molecular evolutionary genetics analysis across computing platforms. Mol. Biol. Evol. 35, 1547-1549. doi: 10.1093/molbev/msy096

Letunic, I., and Bork, P. (2019). Interactive tree of life (iTOL) v4: recent updates and new developments. Nucleic Acids Res. 47, W256-W259.

Mcgoldrick, A., Chanter, J., Gale, S., Parr, J., Toszeghy, M., and Line, K. (2013). Real time PCR to detect and differentiate Campylobacter fetus subspecies fetus and Campylobacter fetus subspecies venerealis. J. Microbiol. Methods 94, 199-204. doi: 10.1016/j.mimet.2013.06.014

Mcmillen, L., Fordyce, G., Doogan, V. J., and Lew, A. E. (2006). Comparison of culture and a novel 5' Taq nuclease assay for direct detection of Campylobacter fetus subspecies. venerealis in clinical specimens from cattle. J. Clin. Microbiol. 44, 938-945. doi: 10.1128/jcm.44.3.938-945.2006

Moolhuijzen, P. M., Lew-Tabor, A. E., Wlodek, B. M., Agüero, F. G., Comerci, D. J., Ugalde, R. A., et al. (2009). Genomic analysis of Campylobacter fetus subspecies: identification of candidate virulence determinants and diagnostic assay targets. BMC Microbiol. 9:86. doi: 10.1186/1471-2180-9-86

OIE Terrestrial Manual (2018). Bovine Genital Campylobacteriosis (Chapter 3.4.4). Available online at: https://www.oie.int/fileadmin/Home/eng/Health standards/tahm/3.04.04_BGC.pdf (Accessed February 17, 2020)

On, S. L., and Harrington, C. S. (2001). Evaluation of numerical analysis of PFGEDNA profiles for differentiating Campylobacter fetus subspecies by comparison with phenotypic, PCR and $16 \mathrm{~S}$ rDNA sequencing methods. J. Appl. Microbiol. 90, 285-293. doi: 10.1046/j.1365-2672.2001.01247.x

Pritchard, L., Glover, R. H., Humphris, S., Elphinstone, J. G., and Toth, I. K. (2016). Genomics and taxonomy in diagnostics for food security: soft-rotting enterobacterial plant pathogens. Anal. Methods 8, 12-24. doi: 10.1039/ c5ay02550h

Richter, M., and Rossello-Mora, R. (2009). Shifting the genomic gold standard for the prokaryotic species definition. Proc. Natl. Acad. Sci. U.S.A. 106, 19126 19131. doi: 10.1073/pnas.0906412106

Schulze, F., Bagon, A., Müller, W., and Hotzel, H. (2006). Identification of Campylobacter fetus subspecies by phenotypic differentiation and PCR. J. Clin. Microbiol. 44, 2019-2024. doi: 10.1128/jcm.02566-05

Shen, W., Le, S., Li, Y., and Hu, F. (2016). SeqKit: a cross-platform and ultrafast toolkit for FASTA/Q file manipulation. PLoS One 11:e0163962. doi: 10.1371/ journal.pone.0163962

Silveira, C. D. S., Fraga, M., Giannitti, F., Macías-Rioseco, M., and Riet-Correa, F. (2018). Diagnosis of bovine genital campylobacteriosis in South America. Front. Vet. Sci. 5:321. doi: 10.3389/fvets.2018.00321

Sprenger, H., Zechner, E. L., and Gorkiewicz, G. (2012). So close and yet so far - molecular microbiology of Campylobacter fetus subspecies. Eur. J. Microbiol. Immunol. 2, 66-75. doi: 10.1556/eujmi.2.2012.1.10

Stamatakis, A. (2014). RAxML version 8: a tool for phylogenetic analysis and post-analysis of large phylogenies. Bioinformatics 30, 1312-1313. doi: 10.1093/ bioinformatics/btu033

Van Bergen, M. A., Dingle, K. E., Maiden, M. C., Newell, D. G., and Van Der Graaf-Van Bloois, L. (2005a). Clonal nature of Campylobacter fetus as defined by multilocus sequence typing. J. Clin. Microbiol. 43, 5888-5898. doi: 10.1128/ jcm.43.12.5888-5898.2005

Van Bergen, M. A., Simons, G., Van Der Graaf-Van Bloois, L., Van Putten, J. P., Rombout, J., Wesley, I., et al. (2005b). Amplified fragment length polymorphism based identification of genetic markers and novel PCR assay for differentiation of Campylobacter fetus subspecies. J. Med. Microbiol. 54, 1217-1224. doi: 10.1099/jmm.0.46186-0

Van Der Graaf-Van Bloois, L., Miller, W. G., Yee, E., Rijnsburger, M., Wagenaar, J. A., and Duim, B. (2014). Inconsistency of phenotypic and genomic 
characteristics of Campylobacter fetus subspecies requires reevaluation of current diagnostics. J. Clin. Microbiol. 52, 4183-4188. doi: $10.1128 / \mathrm{jcm}$. 01837-14

Van Der Graaf-Van Bloois, L., Van Bergen, M. A. P., Van Der Wal, F. J., De Boer, A. G., Duim, B., Schmidt, T., et al. (2013). Evaluation of molecular assays for identification Campylobacter fetus species and subspecies and development of a C. fetus specific real-time PCR assay. J. Microbiol. Methods 95, 93-97. doi: 10.1016/j.mimet.2013. 06.005

Wang, G., Clark, C. G., Taylor, T. M., Pucknell, C., Barton, C., Price, L., et al. (2002). Colony multiplex PCR assay for identification and differentiation of Campylobacter jejuni, C. coli, C. lari, C. upsaliensis, and C. fetus subspecies fetus. J. Clin. Microbiol. 40, 4744-4747. doi: 10.1128/jcm.40.12.4744-47 47.2002
Xie, Z., and Tang, H. (2017). ISEScan: automated identification of insertion sequence elements in prokaryotic genomes. Bioinformatics 33, 3340-3347. doi: 10.1093/bioinformatics/btx433

Conflict of Interest: The authors declare that the research was conducted in the absence of any commercial or financial relationships that could be construed as a potential conflict of interest.

Copyright () 2020 Abdel-Glil, Hotzel, Tomaso and Linde. This is an open-access article distributed under the terms of the Creative Commons Attribution License (CC BY). The use, distribution or reproduction in other forums is permitted, provided the original author(s) and the copyright owner(s) are credited and that the original publication in this journal is cited, in accordance with accepted academic practice. No use, distribution or reproduction is permitted which does not comply with these terms. 